\title{
Refractory HHV-6 encephalitis in a patient treated with anti-CD20 monoclonal antibody therapy
}

\author{
Jennifer C Massey ${ }^{1-3 *}$ \\ ${ }^{1}$ Department of Neurology, St Vincent's Applied Medical Research, Vincent's Hospital Sydney, Australia \\ ${ }^{2}$ Department of Haematology and BM Transplantation, St Vincent's Applied Medical Research, Vincent's Hospital Sydney, Australia \\ ${ }^{3}$ Blood, Stem Cells and Cancer Research Programme, St Vincent's Applied Medical Research, Vincent's Hospital Sydney, Australia
}

\section{Introduction}

Human herpes virus 6 (HHV-6) is an infectious cause of encephalitis, rarely identified following allogeneic haematopoietic stem cell transplantation (alloHSCT). The typical symptoms are memory loss, deteriorating conscious state and seizures, which reflect limbic pathology and may be demonstrated by MRI. To date only 9 cases reported in non-alloHSCT patients [1-3]. This report describes the clinical, radiological and pathological features of a patient with HHV6 encephalitis who was treated with chlorambucil and obinutuzumab (a CD-20 monoclonal antibody) for chronic lymphocytic leukaemia (CLL), where HHV-6 was detected in cerebrospinal fluid (CSF) and brain tissue via nucleic acid detection. The case highlights the importance of considering HHV-6 as a cause of encephalitis in patients undergoing low or intermediate immunosuppression, of particular relevance given the introduction of $\mathrm{B}$ cell therapies for multiple sclerosis [4].

\section{Case Report}

The patient is a 72 year old farmer who was receiving chemotherapy with chlorambucil for CLL. Three months prior to presentation he had adjuvant treatment with 3 cycles of Obinutuzumab; a monoclonal CD20 antibody dosed monthly, as part of a clinical trial. Obinutuzumab was ceased due to systemic side effects including fever, sweats, weight loss and malaise, and single agent therapy with Chlorambucil was continued. Despite cessation of Obinutuzumab, the symptoms continued, and were accompanied by apathy, generalised weakness and muscular atrophy, deteriorating cognition and occasional visual hallucinations. There was no headache. During an admission to hospital for investigation of the above symptoms, two right upper limb focal motor seizures occurred with subsequent, secondary generalisation and status epilepsy requiring intensive care. Magnetic resonance imaging (MRI) showed areas of T2 and FLAIR high signal within the superior left frontal lobe and right parafalcine region, without gadolinium enhancement or restricted diffusion (Figure 1). An EEG demonstrated poorly developed background rhythms occasionally reaching $8 \mathrm{~Hz}$, symmetric throughout the recording and no focal seizure activity after treatment. A lumbar puncture showed a raised CSF protein of $2.46 \mathrm{~g} / \mathrm{L}$ $(0.15-0.45 \mathrm{~g} / \mathrm{L})$, normal glucose and a CSF pleocytosis of $165 \times 10^{\wedge} 5 / \mathrm{L}$ mononuclear cells.

Overall the clinical picture was consistent with encephalitis. Lymphoproliferative, infective and paraneoplastic/autoimmune causes were considered. Initial CSF culture and viral PCR for HSV, VZV and enterovirus were negative. John Cunningham Virus (JCV)
PCR was repetitively negative on serial CSFs. Flow cytometry and cytology excluded infiltration with CLL but showed an extraordinary predominance of CD4 T- lymphocytes. Screening for paraneoplastic antibodies (anti-neuronal antibodies including VGKC, NMDA-R) in CSF and serum was negative.

The patient deteriorated over the next two weeks with rapidly progressive cognitive impairment to the point of becoming aphasic, with recurrent visual hallucinations and intermittent focal motor seizures of both upper limbs independently. A brain biopsy of the left frontal lesion was undertaken (Figure 2). The specimen included grey and white matter. Findings were unusual, with a predominance of small, reactive and perivascular CD4+ lymphocytes in the absence of a specific pathology. Demyelination and necrosis were absent and axons and neurons were preserved. There was neither neuronophagia, viral inclusions nor bizarre astrocytes. CD8 T-lymphocytes were occasionally found in the parenchyma and there were rare B-lymphocytes. Biopsy effectively excluded lymphoma, with viral infection or paraneoplastic disease the likely differential aetiology and a trial of intravenous steroids was administered without effect. CSF was checked for HHV6 and HHV8 using PCR on two occasions. Both CSF specimens were positive for HHV6. Brain tissue was also positive for HHV6 by PCR. Treatment with foscarnet was commenced, and subsequently changed to ganciclovir due to acute kidney injury. Antiviral agents were continued for a period of 21 days and anticonvulsants tapered to maintenance doses. Serial MRI scans showed resolution of the T2-FLAIR high signal change involving the posterior frontal lobes bilaterally. A left caudate nucleus lesion had developed in the interim. The patient improved cognitively and gradually regained sufficient strength to walk and was discharged 6 months after the original presentation. Serial CSF examinations demonstrated that anti-viral treatment suppressed HHV-6 to undetectable levels, but withdrawal of treatment resulted in recurrent encephalitis with clinical deterioration and the re-appearance of HHV-6 DNA in CSF. As such, he remains on maintenance treatment with oral valganciclovir.

\section{Discussion}

HHV-6 reactivation is common among patients post alloHSCT and is known to cause limbic encephalitis, but rare in patients who are immunocompetent or have received alternate treatments for

${ }^{\star}$ Correspondence to: Jennifer C Massey, Neurologist, St Vincent's Hospital, Sydney, Australia, E-mail: jennifer.massey@hotmail.com

Received: June 20, 2018; Accepted: June 25, 2018; Published: June 30, 2018 

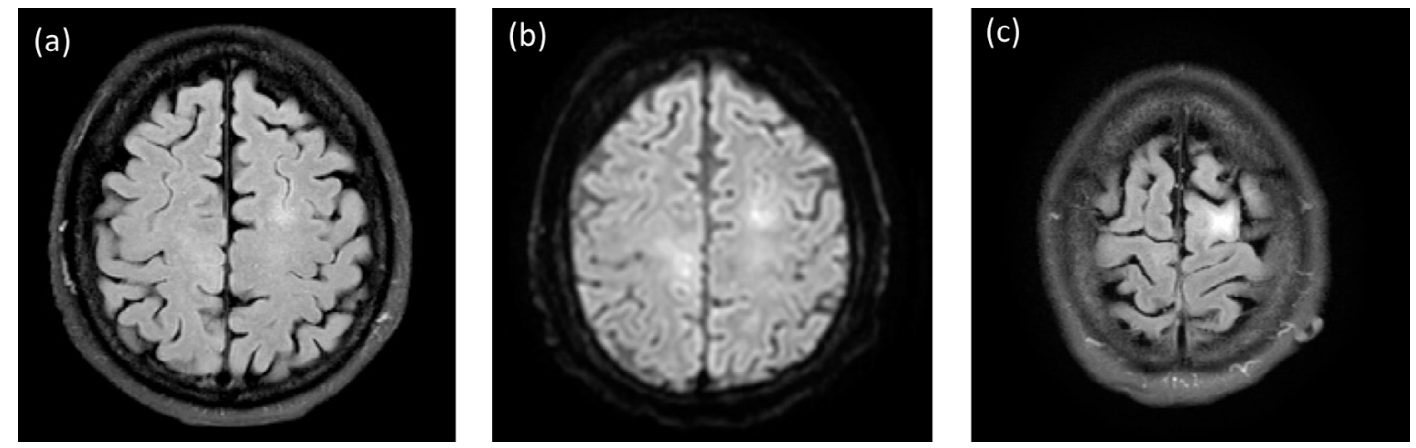

Figure 1. (a) Axial FLAIR image demonstrating hyperintensity of the left superior frontal lobe and right parafalcine lesion. (b) and (c) Pre-biopsy DWI B1000 and axial FLAIR demonstrating progression of both lesions
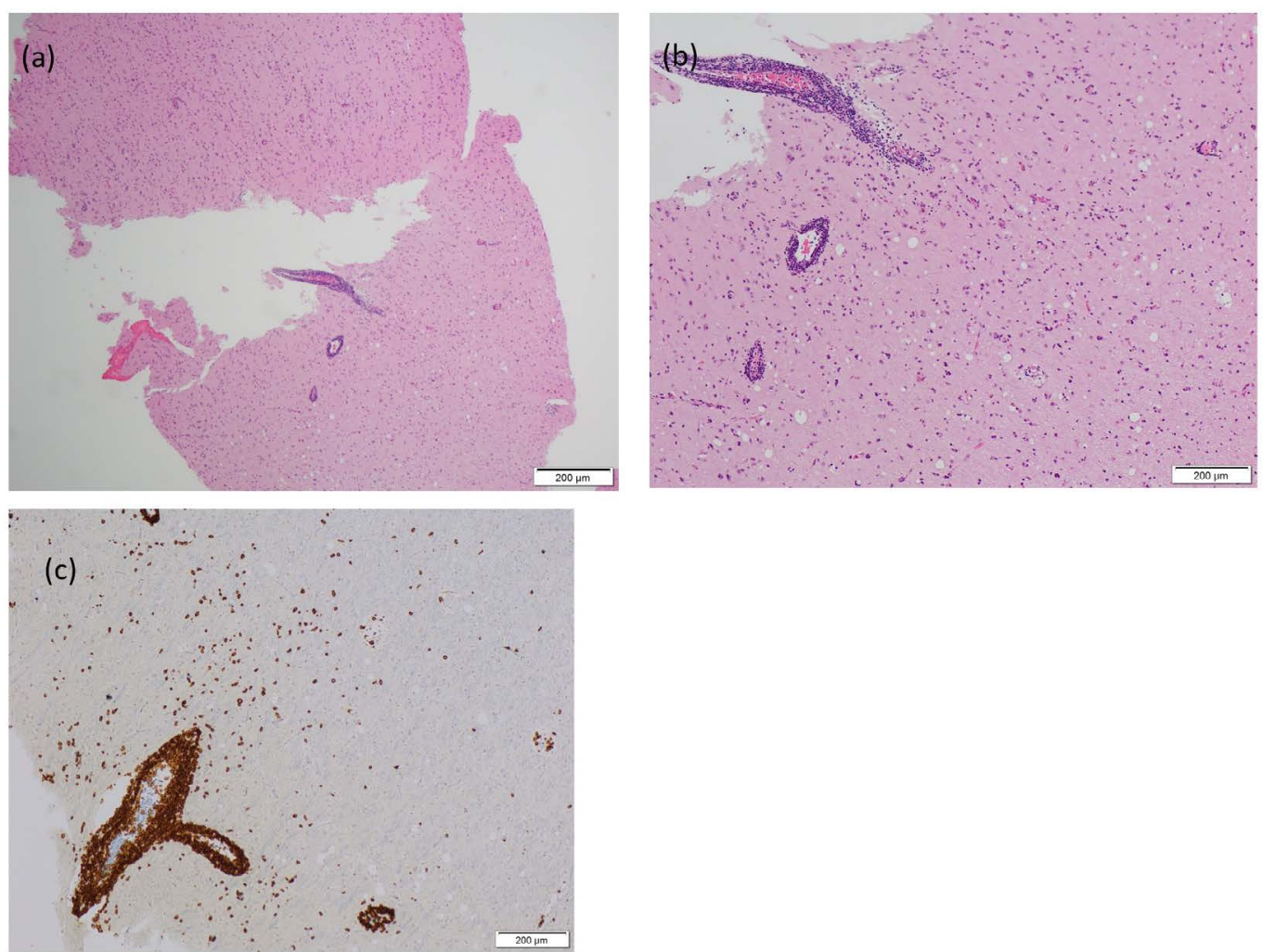

Figure 2. (a) H\&E x40: Brain cortex and white matter with prominent perivascular lymphocytic infiltrate. (b) H\&E x100: Prominent perivascular lymphocytic infiltrate and smaller numbers of lymphocytes in the brain parenchyma. (c) CD3 immunostain x100: Lymphocytes (perivascular and in the brain parenchyma) are mainly reactive T- lymphocytes

haematological malignancies. To date only 9 cases have been reported in the English literature of patients who have not had preceding AHSCT or solid organ transplant. Development of HHV6 encephalitis in the context of anti-CD20 mAb use is particularly relevant given the recent launch of Ocrelizumab, a humanised monoclonal antibody against CD20, for the use in multiple sclerosis [4]. Awareness of rare, infective causes of new MRI lesions in patients treated with anti-CD20 therapy is vital for neurologists planning to initiate this treatment in an MS patient cohort.

The driving factors for HHV6 reactivation remain poorly understood, and there is additional debate as to whether HHV-6 encephalitis results from viral reactivation in immunocompetent patients or primary infection. In alloHSCT reactivation of HHV-6 is observed in 30-70\% of patients between weeks 2-4 [5]. Encephalitis develops concomitant to peak serum replication, with a correlation between higher HHV-6 serum viral load and development of encephalitis. While there is a paucity of evidence for HHV-6 infection of neuronal cells, the virus is known to display tropism for astrocytes in the hippocampus [5]. It is hypothesised that the ability of HHV6 to evade host immune responses in the context of moderate intensity immunosuppression (including monoclonal antibodies) and associated viral down-regulation of cytokines including IL-2 may be adequate to allow viral reactivation resulting in infection of microglia, astrocytes and oligodendrocytes. It is therefore proposed that the CNS may act as a reservoir for HHV-6 infection, even in the absence of viral replication in immunocompromised individuals. This would explain the recurrence of CSF PCR positivity seen in our patient repeatedly after weaning anti-viral medication.

Outcomes in AHSCT cases are invariably poor, with a reported mortality rate of roughly $25 \%$ of patients. Of the 'immunocompetent' 
cases described in the literature, all patients survived following antiviral treatment, with the majority experiencing a protracted recovery and mild ongoing neurocognitive deficits [1-3]. No controlled trial to evaluate the efficacy of anti-viral agents has been performed, although infectious disease guidelines including Clinical Practice Guideline by the Infectious Disease Society of America and the European Conference of Infections in Leukaemia all recommend foscarnet or ganciclovir as first-line therapies for HHV-6 encephalitis. Duration of therapy is unclear but principles of 21 days therapy as per HSV encephalitis are typically prescribed, or until serial CSF PCR testing is negative. Our patient relapsed multiple times on attempted weaning of anti- viral medications, and continued to respond to re-treatment, creating challenges in anti-infective treatment in a condition where CNS viral clearance appears transient. This case of HHV6 encephalitis following moderate intensity immunosuppression should serve as a reminder to clinicians to consider testing for this virus in other patients presenting with similar clinical features beyond patients post AHSCT, particularly in MS patients where use of anti-CD20 therapy is likely to grow in the near future.

\section{References}

1. Shahani L (2014) HHV-6 encephalitis presenting as status epilepticus in an immunocompetent patient. BMJ Case Rep 1-3. [Crossref]

2. Isaacson E, Glaser CA, Forghani B, Amad Z, Wallace M, et al. (2005) Evidence of human herpesvirus 6 in 4 immunocompetent patients with encephalitis. Clin Infect Dis 40: 890-3. [Crossref]

3. De Simone M, Muccio CF, Megna AS, Esposito G (2013) Human herpes virus 6 encephalitis in immunocompetent patient: an unusual neuroradiological presentation. Clin Neurol Neurosurg 115: 2219-22. [Crossref]

4. Hauser SL, Bar-Or A, Comi G, Giovannoni G, Hartung HP, et al. (2017) Ocrelizumab versus Interferon Beta-1a in Relapsing Multiple Sclerosis. N Engl J Med 376: 221-34. [Crossref]

5. Ogata M, Fukuda T, Teshima T (2015) Human herpesvirus-6 encephalitis after allogeneic hematopoietic cell transplantation: What we do and do not know. Bone Marrow Transplant 50: 1030-6. [Crossref]

Copyright: (C2018 Massey JC. This is an open-access article distributed under the terms of the Creative Commons Attribution License, which permits unrestricted use, distribution, and reproduction in any medium, provided the original author and source are credited. 